\title{
Encuesta Nacional de Tendencias de Fitness en España para 2022 National Survey of Fitness Trends in Spain for 2022
}

\author{
*Oscar L Veiga, **Manel Valcarce-Torrente, *Alejandro Romero-Caballero \\ *Universidad Autónoma de Madrid (España), **Universidad Internacional de Valencia (España)
}

\begin{abstract}
Resumen. Esta es la sexta edición de las Encuestas Nacionales de Tendencias de Fitness en España, que reproducen de forma anual la metodología de las encuestas internacionales promovidas por el Colegio Americano de Medicina del Deporte (ACSM). El objetivo del presente estudio es identificar las principales tendencias en el sector del fitness español mediante la presentación de los resultados de la encuesta realizada para 2022, así como comparar los resultados con los obtenidos en las encuestas nacional e internacional de 2021. Además, también se analizó el posible impacto del COVID-19 en la incorporación de tecnología en el sector del fitness en España. La encuesta fue enviada electrónicamente a un total de 6551 profesionales relacionados con el sector del fitness recogiéndose un total de 520 respuestas (tasa de respuesta de 7.9\%), de las cuales un 27.3\% fueron mujeres. Las cinco tendencias más relevantes para 2022 fueron, por ese orden: «contratación de profesionales certificados», «entrenamiento funcional de fitness», «entrenamiento personal en pequeños grupos», «entrenamiento personal» y «programas de ejercicio y pérdida de peso». Las tendencias en el top-20 en España para 2022 fueron muy congruentes con las de 2021, tanto en el número de coincidencias (18 tendencias comunes) como en las posiciones ocupadas (dentro del top10 hubo 9 tendencias comunes a ambas encuestas). Al igual que en años anteriores, se produjo una mayor discrepancia entre la encuesta española para 2022 y la encuesta internacional de 2021, con sólo 13 tendencias comunes dentro del top-20 y únicamente dos coincidencias en el top-10. Estos hallazgos vuelven a confirmar, en consonancia con ediciones anteriores, la estabilidad de las tendencias en el sector del fitness español y la progresiva divergencia con las tendencias internacionales.

Palabras clave: encuesta española fitness, tendencias fitness, industria fitness, encuesta ACSM.
\end{abstract}

\begin{abstract}
This is the sixth edition of the National Surveys of Fitness Trends in Spain, which annually reproduces the methodology of the worldwide surveys endorsed by the American College of Sports Medicine (ACSM). The objective of this study is to identify the main trends in the Spanish fitness sector by presenting the results of the Spanish survey for 2022, as well as comparing the results with those obtained in the national and international surveys for 2021. In addition, the possible impact of COVID-19 on the incorporation of technology in the fitness sector in Spain was also analyzed. The survey was electronically sent to 6551 professionals related to the fitness sector, collecting a total of 520 responses (response rate 7.9\%), of which $27.3 \%$ were women and $72.7 \%$ were men. The five most relevant trends for 2022 were, in that order: «employing certificate fitness professional», «functional fitness training», «personal training in small groups», «personal training» and «exercise and weight loss programs». The trends in the top-20 in Spain for 2022 were very congruent with those of 2021, both in the number of coincidences (18 common trends) and in the positions occupied (9 common trends within the top-10). As in previous years, there was a greater discrepancy between the Spanish survey for 2022 and the worldwide survey for 2021, with only 13 common trends within the top-20 and only two coinciding trends in the top-10. These findings confirm again, in accordance with previous editions, the stability of trends in the Spanish fitness sector and the progressive divergence with worldwide trends.
\end{abstract}

Key words: Spanish fitness survey, fitness trends, fitness industry, ACSM survey.

\section{Introducción}

Desde hace 15 años el Colegio Americano de Medicina del Deporte (ACSM: American College of Sports Medicine) viene realizando anualmente la encuesta mundial sobre tendencias del fitness (Thompson, 2006-2020). Mediante estas encuestas, que engloban las respuestas de miles de profesionales del sector procedentes de

Fecha recepción: 09-06-21. Fecha de aceptación: 02-12-21

Oscar L Veiga

oscar.veiga@uam.es diferentes países, se pretende conocer de forma prospectiva cuáles serán las principales tendencias a nivel global (Thompson, 2020). Los resultados obtenidos permiten anticipar cuáles de ellas podrían tener impacto en la industria de acuerdo con la opinión de las personas encuestadas y, por lo tanto, ayudan a tomar importantes decisiones estratégicas y comerciales para su desarrollo futuro (Thompson, 2020).

Con el propósito de analizar el contexto particular en busca de singularidades del sector, España fue el primer país del mundo en replicar las encuestas internacionales del ACSM a nivel nacional. Desde 2017 se han publicado cinco ediciones (de la Cámara et al., 2020; 
Veiga et al., 2017, 2018, 2019, 2020) que, junto a la presente, permiten observar la evolución de las tendencias durante los últimos seis años, permitiendo identificar cómo muchas de las tendencias presentes en las encuestas internacionales lo son también en España, mientras que otras aparecen como tendencias singulares del contexto español o bien, por el contrario, lo son exclusivamente en el contexto internacional (Veiga et al., 2020). Así mismo, permite observar si las divergencias entre los resultados de ambas encuestas se atenúan o incrementan.

Tras la réplica pionera en España, en los últimos años diversos investigadores han realizado y publicado encuestas similares en otras regiones del mundo entre las que destacan la encuesta europea (Batrakoulis, 2019), China (Li et al., 2018), Brasil y Argentina (Amaral \& Palma, 2019), México (Gómez et al., 2020), Grecia (Batrakoulis et al., 2020), Portugal (Franco et al., 2021) y Colombia (Valcarce-Torrente et al., 2021). Así mismo, también se han realizado estudios que comparan los resultados de las encuestas regionales (Kercher, 2018; Kercher et al., 2019 y 2021; Vorobiova, 2019). La proliferación de encuestas en diferentes lugares del mundo replicando la metodología del ACSM evidencia el creciente interés por la investigación de las tendencias del fitness. Del mismo modo, pone de manifiesto el potencial y la relevancia que pueden tener los resultados obtenidos en estas encuestas para conocer las similitudes en el ámbito trasnacional y las diferencias interregionales de las tendencias en el sector del fitness, permitiendo tener información que pueda orientar de una forma más precisa a los agentes del sector de cada región específica en la toma de decisiones adecuadas (Veiga et al., 2020).

Por otro lado, la crisis sanitaria derivada de la pandemia del virus SARS-CoV-2 (COVID-19) ha tenido un impacto económico negativo muy elevado en el sector del fitness derivado del cierre de los centros de entrenamiento durante semanas (Valcarce-Torrente y García-Fernandez, 2021). Así mismo, como señalanVeiga et al. (2020), tras su progresiva reapertura, las limitaciones de aforo, las medidas de higiene o el miedo al contagio han podido tener un efecto determinante en relación con el tipo de servicios de fitness ofertados y consumidos durante los últimos meses. Especialmente en la relativo a la digitalización de los servicios y el uso de plataformas tecnológicas y aplicaciones móviles para la prestación de nuevos servicios bajo un formato online o virtual (clases, entrenamiento personal o asesoramiento online, entre otros).
Teniendo en cuenta todo lo anterior, el presente estudio ha tenido como objetivo analizar las tendencias del fitness en el contexto español para el año 2022 a través de una encuesta a profesionales del sector, así como comparar los resultados obtenidos con los de la encuesta nacional e internacional del año 2021. Además, este año se ha pretendido analizar cómo ha influido la pandemia del COVID-19 en la incorporación de tecnología en el sector del fitness.

\section{Material y métodos}

El presente estudio replica la metodología de las Encuestas Internacionales sobre Tendencias en Fitness desarrolladas por el ACSM, que vienen realizándose anualmente desde 2006. Mediante las mismas son encuestados los profesionales del sector del fitness cuyo contacto está disponible en las bases de datos del ACSM. El cuestionario, implementado mediante la plataforma SurveyMonkey, es enviado a un gran número de profesionales alrededor del mundo (un total de 75,383 en la última encuesta publicada). La lista de tendencias a evaluar en cada encuesta se selecciona a partir de los resultados de la encuesta anterior, incorporando además algunas tendencias potencialmente emergentes identificadas por el equipo editorial de la ACSM's Health \& Fitness Journal. Cada una de ellas es valorada mediante una escala tipo Likert, tal y como se describirá a continuación. En España la realización de la encuesta está promovida por la Universidad Autónoma de Madrid y la Consultora Deportiva Valgo.

Para la selección de las tendencias a incorporar en la encuesta se incluyeron las 29 primeras tendencias por orden de puntuación de la encuesta nacional para el año 2021. Además, también se incluyeron 7 tendencias que ocuparon posiciones entre la 29 y la 44 en la encuesta española del año 2021 y que estaban dentro de la propuesta del ACSM para el año 2022 según información privada proporcionada por sus autores. Así mismo, se recuperaron 3 tendencias que habían sido propuestas en algún momento en encuestas españolas anteriores y que también se encontraban en la propuesta de tendencias del ACSM para el año 2022. De forma similar, se incorporaron 7 nuevas tendencias recogidas en la propuesta del ACSM para el año 2022. Igualmente, se incorporó una tendencia presente en la encuesta australiana de 2021 («servicios de fitness inclusivo») y se mantuvo de la encuesta pasada la tendencia «influencers/youtubers/ bloggers de fitness» pese a haber tenido una posición baja (\#42) por considerarse que los efectos de la 
pandemia del COVID-19 podrían haber incrementado la su relevancia. Finalmente, se descartaron dos tendencias presentes en la propuesta de la ACSM para el año 2022: «Pilates», al haberse vuelto poco relevante en la encuesta española de 2021 (posición 34) y «programas de incentivos a trabajadores», por no considerarse una tendencia relevante en el contexto español. En total, 48 potenciales tendencias fueron incorporadas a la encuesta española (Tabla 1).
Del mismo modo que en las encuestas internacionales, para valorar la relevancia de las tendencias potenciales se utilizó una escala tipo Likert con valores de 1 (menor probabilidad de ser una tendencia) a 10 (mayor probabilidad de ser una tendencia). Además, se recogió información relativa al sexo, edad, comunidad autónoma de residencia, número de años de experiencia en el sector, ámbito del fitness en el que trabaja, ocupación y situación laboral, así como el lugar principal de trabajo y el nivel de ingresos anuales de cada uno de los encuestados. La encuesta fue realizada en la plataforma Google Forms y enviada electrónicamente, a un total de 6751 profesionales relacionados con el sector del fitness. La tasa de respuesta fue de un 7.9\% con un total de 520 encuestas completadas, similar a la conseguida en las últimas dos encuestas españolas (de la Cámara et al., 2020; Veiga et al., 2020). Se obtuvieron respuestas provenientes de todas las comunidades autónomas españolas, y también de las ciudades autónomas de Ceuta y Melilla. Aunque se trata de una muestra incidental sin selección aleatoria, la estimación de error muestral bajo supuestos de muestreo aleatorio simple, población infinita, nivel de confianza del 95\% y $\mathrm{P}=\mathrm{Q}$ sería de \pm 3.1 $\%$. La encuesta se lanzó el 6 de junio de 2021 y permaneció abierta cuatro sema- 
nas, hasta el 5 de julio del mismo año. Al modelo de cuestionario habitualmente utilizado en las encuestas anteriores se le añadieron un total de 8 preguntas adicionales tratando de recopilar información sobre aspectos relativos al impacto que la COVID-19 esté pudiendo tener sobre la incorporación de tecnología en el sector del fitness. De esta forma 6 preguntas interpelaban sobre la existencia antes y después de la pandemia de sesiones de entrenamiento online (en vivo y bajo demanda), la existencia de servicios de entrenamiento online y sesiones de entrenamiento virtual, así como sobre el uso de app por parte de los centros (tanto para la gestión de aforos como para la programación de entrenamientos). Así mismo, 2 preguntas adicionales investigaban la percepción de los encuestados sobre la extensión del uso de wearables y aplicaciones móviles entre los usuarios para controlar o dirigir sus entrenamientos.

Para identificar las tendencias más relevantes para el año 2022 se calculó la media de las puntaciones obtenidas para cada tendencia potencial, ordenándolas posteriormente de mayor a menor. En este artículo se presentan las 20 tendencias que obtuvieron mayor puntuación. Como en los estudios anteriormente publicados, se discuten los resultados obtenidos contrastándolos con la encuesta nacional de tendencias en fitness para el año 2021, así como con la encuesta internacional para el año 2021, es decir, la última disponible durante la redacción del presente manuscrito. Así mismo, también se presentan los resultados obtenidos en relación al impacto que la COVID-19 esté pudiendo tener sobre la incorporación de tecnología en el sector del fitness, el uso de tecnología vestible (wearables) y aplicaciones móviles para el control del entrenamiento.

\section{Resultados}

Como se puede observar en la tabla 2, relativa a las características principales de los encuestados, la mayoría de los respondedores fueron hombres mientras que las mujeres supusieron un $27.3 \%$ de la muestra, que cubrió un amplio rango de edades, encontrándose el grupo mayoritario de respondedores en la franja de «entre 22 y los 34 años» y «entre los 35 y los 44 años (37.5.7\% y $33.7 \%$ respectivamente). Más de la mitad de los encuestados tiene una experiencia de más de 10 años trabajando en el sector (57.3 \%) y el $47.1 \%$ desarrolla su labor profesional dentro del sector del fitness comercial. Un $86.1 \%$ de los encuestados son trabajadores en activo a tiempo completo o a tiempo parcial,

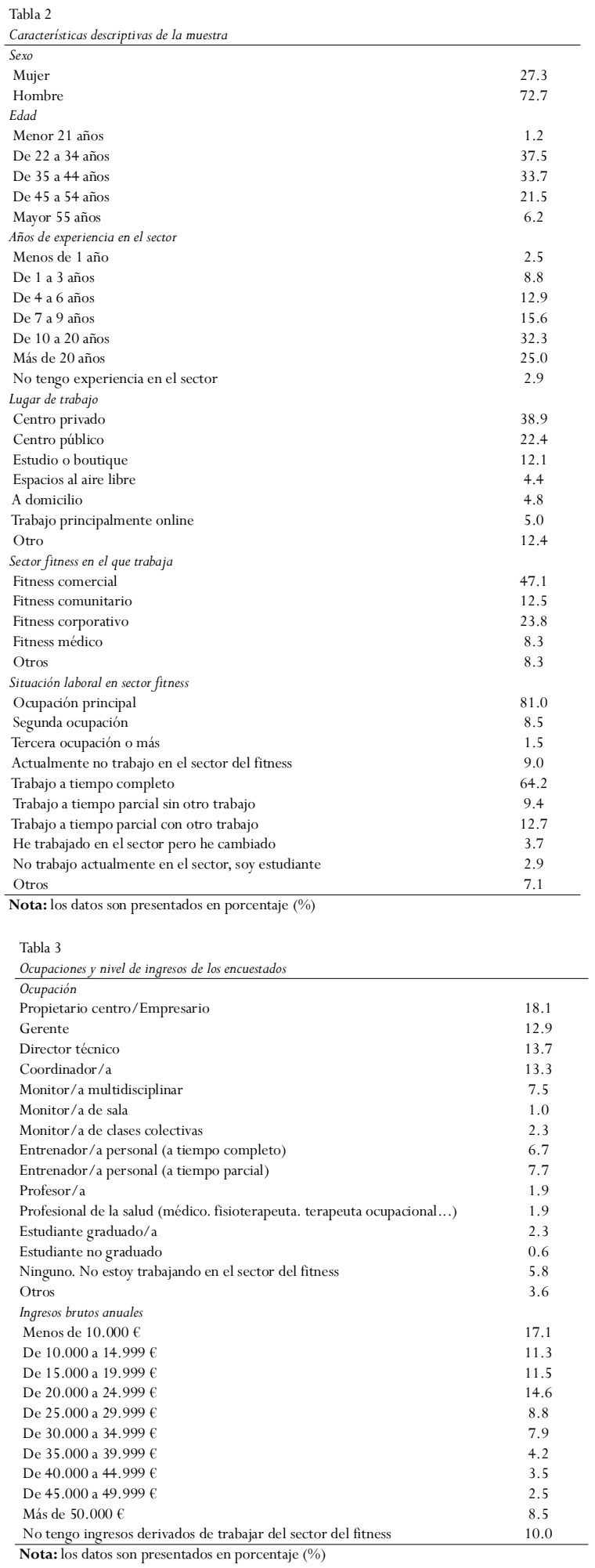

mientras que el 13.9\% restante manifiesta otras situaciones (estar en paro, cambio de sector u otros). Para el $81 \%$ su trabajo en el sector del fitness supone su ocupación principal. Por otro lado, la mayor parte de los encuestados declara trabajar en centros privados de fitness (38.9\%), un $22.4 \%$ en centros de gestión pública, un $12.1 \%$ en estudios o boutiques, un $5 \%$ declara trabajar principalmente online, mientras que el resto afirma hacerlo en otro tipo de centros o lugares. Un 58\% de la muestra ocupa cargos intermedios o de responsabilidad 


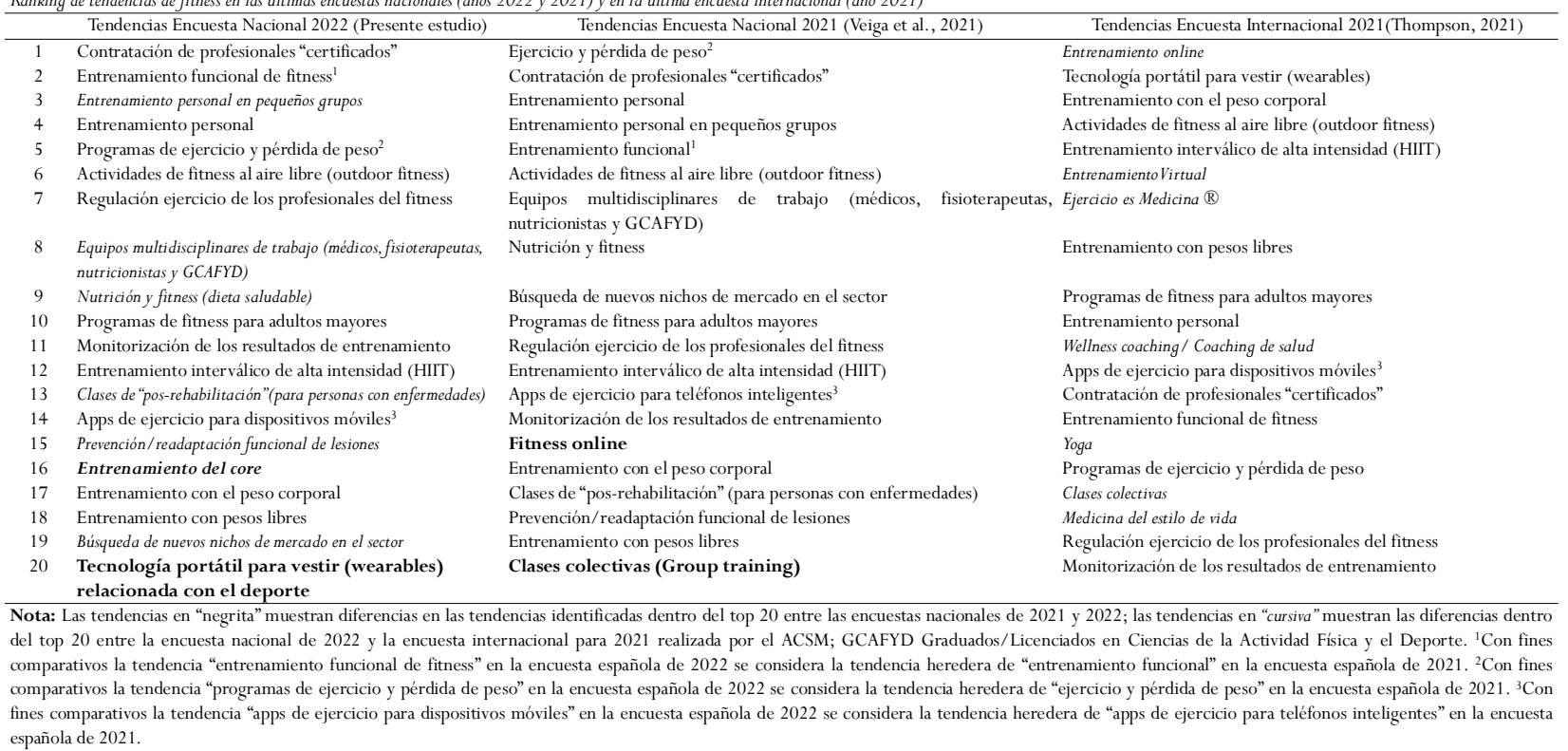

tales como propietario del centro, gerente, director técnico o coordinador, y la mayoría de los profesionales $(54.5 \%)$ declara tener unos ingresos inferiores a 25000 euro/año (Tabla 3).

En la tabla 4 se pueden observar las 20 tendencias principales identificadas para los años 2021 y 2022 en España, además de las identificadas en la encuesta internacional para el año 2021. Los resultados de la encuesta para 2022 muestran una gran congruencia con los de la encuesta del año anterior, con únicamente 2 tendencias diferentes. Las nuevas tendencias que aparecen para el año 2022 son «entrenamiento del CORE»y «tecnología portátil para vestir «wearables» relacionada con el deporte», desplazando del top-20 a las tendencias «fitness online» y «clases colectivas (group training)» de la encuesta de 2021. Además, encontramos una gran coincidencia también en cuanto a las posiciones ocupadas. Las 6 primeras tendencias en las encuestas nacionales para 2022 y 2021 coinciden y, dentro del top-12, 11 de las tendencias también aparecen en ambas encuestas. En el top-5 para el año 2022, el primer puesto lo ocupa la tendencia «contratación de profesionales certificados», seguido por «entrenamiento funcional de fitness», «entrenamiento personal en pequeños grupos», «entrenamiento personal»y «programas de ejercicio y pérdida de peso».

Por el contrario, solo 13 tendencias de la encuesta nacional para 2022 coinciden con las del top-20 de la última encuesta internacional. Las 7 tendencias no coincidentes (que aparecen en cursiva dentro de la tabla 4) son: «entrenamiento personal en pequeños grupos», «equipos multidisciplinares de trabajo (médicos, fisioterapeutas, nutricionistas y GCAFYD)», «nutrición y fitness», «clases de pos-rehabilitación (para personas con enfermedades)», «prevención/readaptación funcional de lesiones», «entrenamiento del core»y «búsqueda de nuevos nichos de mercado en el sector». Así mismo, las tendencias de la última encuesta internacional que no aparecen en la encuesta española para 2022 son: «entrenamiento online», «entrenamiento virtual», «Ejercicio es Medicina ${ }^{\circledR} »$, «health/wellness coaching», «yoga», «clases colectivas» y «medicina del estilo de vida». Comparando las primeras posiciones de la encuesta nacional para 2022 con la encuesta internacional para 2021 sólo la tendencia «actividades de fitness al aire libre (outdoor fitness) coincide dentro del top-5 y, si ampliamos la selección hasta las 10 primeras posiciones, sólo se incorpora la tendencia «programas de fitness para adultos mayores», sumando un total de 2 coincidencias en el top- 10 .

Finalmente, la tabla 5 refleja el impacto del COVID19 en la incorporación de tendencias tecnológicas en el sector del fitness. Como se puede apreciar, la proporción de encuestados que afirma la existencia de sesiones de entrenamiento online y en directo (disponibles en streaming para los usuarios) fue mucho mayor después de la pandemia (71.3\%) que antes de la misma (32.8\%). Este fenómeno se repite en relación con la existencia de sesiones de entrenamiento online grabadas y disponibles bajo demanda para los usuarios (70.1vs 40.6\%), la utilización de aplicaciones para la gestión de aforos (64.8 vs $49.5 \%$ ) y la existencia de servicios de entrenamiento personal online (30.7 vs $17.6 \%$ ). Sin embargo, no se aprecian grandes diferencias entre antes y después de la pandemia en el uso de aplicaciones para el desarrollo de entrenamientos de los usuarios o el uso de 
clases virtuales presenciales (sin monitor y únicamente soportadas por la proyección de material audiovisual en la sala de entrenamiento) que, según los resultados, están presentes aproximadamente en uno de cada dos centros.

Además de la información presentada en la tabla 5, se recogió información respecto al uso de tecnología wearables y el uso apps de entrenamiento por parte de los usuarios de fitness. Los resultados obtenidos mostraron que sólo en torno a un tercio de los encuestados (28.9\%) piensa que más del $40 \%$ de los usuarios usa tecnología wearable e, igualmente, solo en torno a un tercio $(32.5 \%$ ) piensa que más de $50 \%$ de los usuarios usa apps para entrenar o monitorizar su entrenamiento.

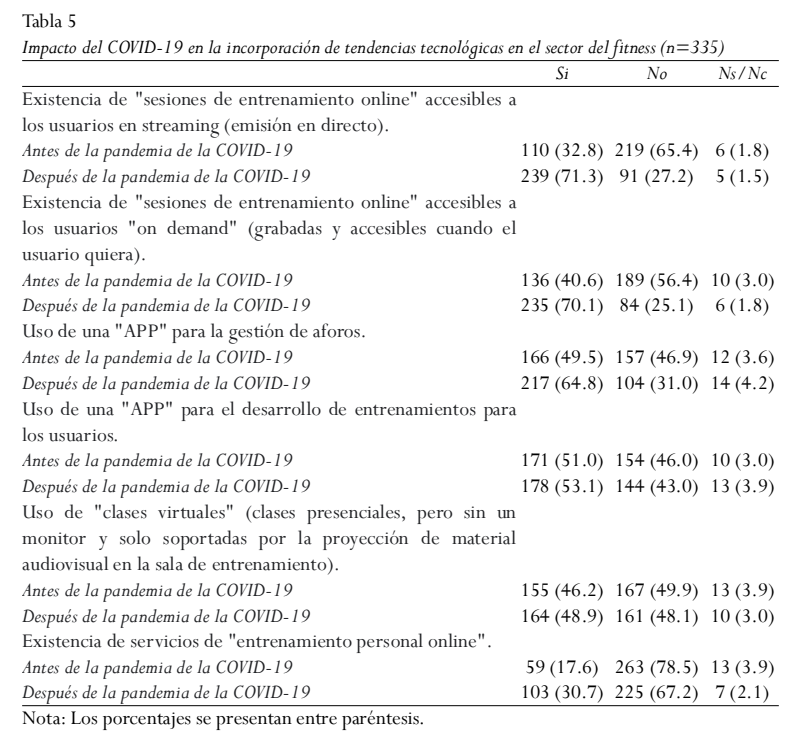

\section{Discusión}

El objetivo del presente estudio ha sido identificar las tendencias del fitness en España para el 2022 y compararlas con las del año anterior tanto en el contexto nacional como en el internacional. Para ello se diseñó una encuesta online que fue distribuida entre un amplio número de profesionales del sector del fitness en España. Los resultados muestran una gran coincidencia en el top-20 entre las tendencias de las encuestas nacionales para 2021 y 2022, tanto en el número de tendencias que aparecen en ambas ediciones (18 tendencias comunes) como en las posiciones ocupadas.

Existen tendencias que incrementan su relevancia con respecto a años anteriores, mejorando 3 o 4 posiciones con respecto al año 2021. Este es el caso de las tendencias «entrenamiento funcional de fitness», «regulación ejercicio de los profesionales del fitness», «monitorización de los resultados de entrenamiento», «clases de pos-rehabilitación», «prevención/readapta- ción funcional de lesiones», «entrenamiento del core» y «tecnología portátil para vestir (wearables) relacionada con el deporte».

Por contraposición encontramos dos tendencias que, aun permaneciendo dentro del top-20, empeoraron claramente con respecto al año anterior. Estas son las tendencias «programas de ejercicio y pérdida de peso», que bajó desde la primera posición en el año 2021 hasta la quinta para 2022, y «búsqueda de nuevos nichos de mercado en el sector», desplomándose desde la novena hasta la décimo novena plaza del ránking. El resto permanecieron aproximadamente en las mismas posiciones que en el año 2021.

En relación con la comparación entre la encuesta nacional de 2022 y la internacional de 2021 sólo aparecen 13 tendencias coincidentes en el top-20, ocupando además posiciones bastante diferentes. Como ocurrió en ediciones anteriores, la «tecnología portátil para vestir (wearables) relacionada con el deporte» parece tener una relevancia mucho menor en la encuesta española, donde aparece en la posición \#20 (tras tres años fuera de las 20 primeras plazas del ranking) que en el contexto internacional donde sigue siendo una de las tendencias mejor situadas (\#2 en el año 2021). Por otro lado, llama la atención que en la encuesta española para 2022 no aparezca el «entrenamiento online», que en 2021 ocupó el puesto 15 en la encuesta española y el primer puesto en la encuesta internacional. Así mismo, al igual que en ediciones anteriores, algunas tendencias relacionadas con la salud como «Ejercicio es Medicina $\left.{ }^{(}\right)$, «wellness coaching/coaching de salud» o «medicina del estilo de vida» son solo relevantes en la encuesta internacional. Por el contrario, algunas tendencias que aparecen en el top-20 de la encuesta nacional para 2022 son exclusivas de la encuesta española y no se incorporan en la propuesta de tendencias de la encuesta internacional. Algunos ejemplos son los «equipos multidisciplinares de trabajo», «nutrición y fitness» o «prevención/readaptación funcional de lesiones». Todo ello parece confirmar la tendencia mostrada durante los últimos años, en los que el número de coincidencias entre la encuesta nacional y la internacional se sitúan entre 13 y 14. A continuación se presentan y discuten las 20 tendencias más relevantes en la encuesta nacional para el año 2022, así como los resultados relativos a la opinión de los encuestados sobre el impacto del COVID19 en la incorporación de tecnología en el sector del fitness.

1. Contratación de profesionales certificados. Esta tendencia, heredera de «personal formado, cualificado y 
experimentado», asciende hasta el primer puesto del ranking tras varios años en las primeras plazas. El recorrido histórico de esta tendencia muestra la relevancia de la adecuada cualificación de los profesionales del sector del fitness en España. Por el contrario, en la encuesta internacional esta tendencia ha experimentado un progresivo descenso, situándose en 2021 en la posición no.13 tras ocupar puestos superiores en años anteriores.

2. Entrenamiento funcional de fitness. Este tipo de entrenamiento está orientado a la mejora de la fuerza muscular, involucrando a su vez contenidos para la optimización del equilibrio, la coordinación y la resistencia, con el principal objetivo de incrementar la funcionalidad cotidiana de las personas, es decir, su capacidad para llevar a cabo actividades de la vida diaria. Esta tendencia, heredera del «entrenamiento funcional», sigue mostrando su relevancia al mantenerse varios años seguidos dentro del top-5 en nuestro país. Además, esta tendencia parece ser mucho más relevante en el contexto nacional español que en el internacional, donde aparece en la posición 14, manteniendo las posiciones intermedias de los últimos años.

3. Entrenamiento personal en pequeños grupos. El entrenamiento personal en pequeños grupos es una alternativa más económica que el entrenamiento personal tradicional. El entrenador trabaja con grupos no superiores a las cinco personas, permitiendo así prestar un servicio personalizado a un precio significativamente inferior. Esta tendencia reafirma su importancia al repetir dentro del top- 5 por segundo año consecutivo. Esta tendencia continúa sin aparecer en el top-20 de la encuesta internacional después de que apareciese por última vez en la encuesta del año 2018.

4. Entrenamiento personal. Esta tendencia hace referencia al entrenamiento personal tradicional, en el que el entrenador dirige a un único cliente. Se mantiene dentro del top-5 en la encuesta nacional, su lugar habitual a excepción del año 2020, en el que bajó hasta la posición no.7. Por el contrario, pierde fuerza en la encuesta internacional, bajando hasta el décimo puesto, el más bajo en el histórico de encuestas -desde 2007-.

5. Programas de ejercicio y pérdida de peso. Esta tendencia aglutina aquellos programas cuyo objetivo sea la creación de un balance energético negativo mediante la combinación de un incremento del gasto energético y una restricción calórica, contribuyendo así a la pérdida de peso. Tras ocupar el primer puesto en la encuesta de 2021, esta tendencia vuelve a ocupar una posición relevante tal y como ha venido haciendo en encuestas espa- ñolas anteriores. Por el contrario, continúa perdiendo relevancia en el contexto internacional, donde ha descendido hasta el puesto no. 16.

6. Actividades de fitness al aire libre (outdoor fitness). Dentro de este grupo se incluyen todas aquellas actividades realizadas en espacios naturales, tales como rutas de senderismo o bicicleta, así como actividades específicamente orientadas al entrenamiento físico como circuitos realizados en parques o espacios similares. Esta tendencia se mantiene en la misma posición que ocupó en la encuesta española del año 2021, mejor posición histórica hasta la fecha, reivindicando su importancia en el contexto de la pandemia de la COVID-19, en el que la realización de actividad física al aire libre se presenta como una alternativa segura para la práctica deportiva al reducir la posibilidad de contagio respecto a las instalaciones de interior. El mismo fenómeno en relación con esta tendencia se ha observado en la encuesta internacional, en la que en el año 2021 ocupó la cuarta posición después de ocupar posiciones relativamente bajas (\#13 a \#17) en años anteriores.

7. Regulación ejercicio de los profesionales de fitness. Esta tendencia, que sigue mostrando su relevancia en consonancia con la progresiva regulación de las profesiones del deporte en España -donde 9 de las 17 comunidades autónomas ya disponen de una legislación específica al respecto (COPLEF, 2019)- vuelve a situarse dentro del top-10 tras salir de éste en el año 2021, momento en el que ocupó la posición no.11. En la encuesta internacional esta tendencia sigue ocupando posiciones muy discretas, hecho que se ha repetido desde su inclusión hace cuatro ediciones. En el año 2021 ocupó el puesto 19.

8. Equipos multidisciplinares de trabajo (médicos, fisioterapeutas, nutricionistas y GCAFYD). Esta tendencia, exclusiva de las encuestas españolas al ser propuesta por sus autores, no tiene equivalente en la encuesta internacional y hace referencia al trabajo conjunto y coordinado de profesionales de distintas áreas (medicina, fisioterapia, ciencias del deporte, nutrición, psicología, etc.) con el objetivo de mejorar la salud de las personas, maximizando los beneficios mediante un abordaje multidisciplinar. Desciende una posición con respecto al año pasado, pero sigue manteniéndose dentro del top10, manifestando su relevancia y estabilidad en el contexto nacional.

9. Nutrición y fitness (dieta saludable). Esta tendencia, que se define como el diseño e implementación de planes dietéticos basados en las necesidades personales y orientadas a la salud, se mantiene en posiciones intermedias tras su inclusión el año pasado en la encuesta 
española. Esta tendencia se incluyó en la encuesta española al incorporarse en la encuesta china de 2020 ocupando una posición relevante, pero no tiene equivalente en las encuestas internacionales ni ha sido incorporada en otras encuestas regionales. En china sigue ocupando una posición extremadamente relevante (puesto 2) en el año 2021 (Kercher et al. 2021).

10. Programas de fitness para adultos mayores. Esta tendencia se refiere a programas de ejercicio dirigidos a personas por encima de la edad de jubilación, supervisados por profesionales y adecuados a las características de esta población para que resulten seguros. Hasta el momento ha aparecido en todas las ediciones de la encuesta española ocupando posiciones intermedias, de forma que con esta décima posición se mantiene la tendencia habitual. En la encuesta internacional de 2021 ocupó la novena posición, manteniendo también la relevancia demostrada durante los últimos años, alrededor de posiciones intermedias. Es interesante comprobar que esta tendencia mantiene su importancia tanto en el contexto nacional como en el internacional, lo que reta al sector del fitness a reinventarse para poder ofrecer servicios seguros para una población especialmente vulnerable ante la COVID-19.

11. Monitorización de resultados de entrenamiento. Esta tendencia hace referencia al esfuerzo realizado en la medición y valoración de los efectos producidos por los programas de entrenamiento, así como en la determinación del grado de consecución de los resultados previstos. Este año ha experimentado una subida de 3 posiciones (\#14 vs \#11), que significa un ligero repunte de una tendencia descendente, y se sitúa de nuevo muy por encima de los puestos ocupados en la encuesta internacional, en la que en el año 2021 ocupó la posición $\mathrm{n}^{\mathrm{o}} 20$.

12. Entrenamiento interválico de alta intensidad (HIIT). El HIIT es un tipo de entrenamiento que incluye intervalos de ejercicio de alta intensidad seguidos por periodos cortos de descanso, suponiendo menos de 30 minutos de trabajo en total. En España, esta tendencia ha ido perdiendo popularidad de forma progresiva desde que ocupó el primer puesto del ranking en 2017. Este año se sitúa en la posición $n^{\circ} 12$, exactamente igual que el año pasado. En el contexto internacional, por su parte, sigue manteniéndose dentro del top-5, manifestando de esta forma una tendencia bastante diferente a la del contexto nacional.

13. Clases de pos-rehabilitación (para personas con enfermedades). Esta tendencia hace referencia a programas de ejercicio diseñados específicamente para personas con enfermedades crónicas de salud tales como cáncer, enfermedades cardiovasculares o párkinson entre otras, y se incorporó con esta denominación en la encuesta de 2020 para adaptarla a la terminología de la encuesta internacional. Esta tendencia se ha mantenido en el top20 durante los 3 últimos años, obteniendo su mejor posición en 2020, descendiendo hasta el puesto 17 el año 2021 y situándose en esta posición intermedia para el año 2022. En la encuesta internacional sigue sin entrar dentro del top-20. Pese a la gran evidencia científica en torno a los beneficios de la práctica de actividad física en personas con enfermedades (Thompson et al., 2020) esta tendencia sigue sin mostrar una gran relevancia tras una única aparición en el top-20 internacional en 2019. Tal vez la percepción de riesgo a la hora de prescribir ejercicio en personas con patologías, la escasa formación de los profesionales sanitarios para diseñar entrenamientos o la reducida incorporación de profesionales de ejercicio a hospitales y centros de salud sigan haciendo que esta tendencia no muestre relevancia en el contexto internacional.

14. Apps de ejercicio para teléfonos inteligentes. Dentro de esta tendencia se enmarcan todas aquellas aplicaciones móviles que han ido apareciendo durante los últimos años que incorporan instrucciones de audio y video para desarrollar ejercicio e incluyendo muchas de ellas sistemas de monitorización, seguimiento y feedback. Ha tenido un comportamiento intermitente tanto en las encuestas nacionales como en las internacionales, alternando posiciones intermedias con desapariciones del top20. Para el año 2022 parece mantenerse en una posición similar a la que ocupó la edición pasada en España (\#13), siendo también muy similar al puesto en el que apareció en 2021 en la encuesta internacional (\#12).

15. Prevención/readaptación funcional de lesiones. Hace referencia a programas de ejercicio orientados tanto a la prevención como a la recuperación funcional y el reentrenamiento tras una lesión y superada la fase médica y fisioterapéutica. Esta tendencia sube 3 puestos respecto a la encuesta nacional del año pasado (ocupó la posición no.18). Al ser una tendencia exclusiva de la encuesta española no aparece en las ediciones de la encuesta internacional y no resulta posible comparación alguna.

16. Entrenamiento del core. El entrenamiento del core tiene como objetivo mejorar la estabilidad general del tronco para transferirla a las extremidades. Por lo general incluye ejercicios que involucran a la zona del abdomen, la zona paravertebral o la cadera. Esta tendencia vuelve a aparecer dentro del top-20 tras dos años de 
ausencia en las encuestas nacionales. Por su parte, en la encuesta internacional no aparece desde 2018. Esta nueva irrupción en el top-20 en España tal vez puede deberse al incremento de la popularidad de los programas de entrenamiento online, tanto en directo como bajo demanda, tras la pandemia de la COVID-19. En éstos, uno de los objetivos recurrentes es el trabajo de la zona media del cuerpo mediante ejercicios que no demandan apenas material y pueden llevarse a cabo prácticamente en cualquier sitio, facilitando así su realización dentro del domicilio.

17. Entrenamiento con el peso corporal. El entrenamiento con el peso corporal, que se realiza con el mínimo equipamiento y usando la carga del peso del cuerpo como principal forma de producir sobrecarga muscular, sigue experimentando un progresivo descenso desde los años 2017-2019, en los que permaneció dentro del top-5. En 2020 esta tendencia ocupó la posición n ${ }^{\circ}$ 14, en 2021 la $\mathrm{n}^{\mathrm{o}} 16 \mathrm{y}$, en la presente edición la $\mathrm{n}^{\mathrm{o}} 17$. Esto contrasta con la gran popularidad que mantiene en la encuesta internacional, donde aparece en 2021 en la cuarta posición. Esta discrepancia entre el contexto nacional y el internacional es destacable teniendo en cuenta lo comentado anteriormente en relación con el auge de los programas de entrenamiento en remoto tras la pandemia de la COVID-19, dentro de los cuales tanto el entrenamiento del core como el entrenamiento con el peso corporal es esperable que mostrasen una gran popularidad.

18. Entrenamiento con pesos libres. Esta tendencia sustituyó al «entrenamiento de fuerza» en las encuestas por considerarse que era demasiado genérica, en el año 2019 para la encuesta internacional y el año 2021 para la encuesta española. Hace referencia aquellos entrenamientos orientados a la fuerza que incorporan todo tipo de pesos libres tales como barra, mancuernas, kettlebells, balones medicinales, etc. (Thompson, 2019). Un año más existe una gran diferencia entre las posiciones ocupadas por esta tendencia entre la encuesta internacional de 2021 (\#8) y la encuesta española de 2022 (\#18) que, sin embargo, es muy similar al que ocupó el año pasado (\#19). Esto confirma que desde el cambio de denominación esta tendencia ha mostrado una pérdida de relevancia en España, donde ocupó el primer puesto en 2020, que fue el último año en el que mantuvo su anterior denominación genérica de «entrenamiento de fuerza».

19. Búsqueda de nuevos nichos de mercado. Esta tendencia hace referencia a la búsqueda de nuevos grupos de clientes que puedan ser atraídos al sector del fitness mediante la creación de productos y servicios que satis- fagan sus necesidades específicas. Aunque siempre ha estado presente en el top-20 español, hasta el año 2020 había experimentado un patrón descendente en su evolución. En el año 2021, sin embargo, rompió esta dinámica para volver a situarse dentro del top-10 (\#9) y en esta edición para 2022 ha sufrido un descenso importante (10 puestos), volviendo a situarse en las últimas posiciones del ranking. Pueden pensarse que ese cambio de tendencia en 2021 pudo deberse al influjo de la COVID-19 en la opinión de los encuestados. En las encuestas internacionales esta tendencia no aparece en el top-20 desde 2013.

20. Tecnología portátil para vestir (wearables) relacionada con el deporte. Esta tendencia incluye relojes inteligentes, monitores de actividad, así como textiles que incorporan tecnología GPS o monitores de frecuencia cardiaca, entre otros. Vuelve a aparecer en la encuesta española tras tres años en los que no entró dentro del top-20. No obstante, aún se observa que tiene una relevancia mucho menor en el contexto nacional que en el internacional, donde ha solido ocupar las primeras posiciones del ranking. Así, en el 2021 se situó en la segunda posición de la encuesta internacional mostrando una situación casi opuesta al caso español.

\section{¿Qué queda fuera en la encuesta de 2022?}

Sólo dos tendencias han salido del top-20 en esta edición en comparación con la del año pasado. Una de ellas es el «fitness online», lo que llama la atención si tenemos en cuenta los resultados de la encuesta internacional de 2021, en la que aparece en la primera posición del ranking, y los de la encuesta nacional de 2021, donde entra en el top-20 situándose en el puesto 15. Resulta remarcable no encontrar esta tendencia dentro del top-20 en el contexto derivado de la COVID-19, especialmente propicio para la aparición de tendencias con un componente tecnológico importante. Así mismo, también sale del top-20 la tendencia relativa a «clases colectivas», que ya había perdido relevancia situándose en el puesto 20 en el año 2021. Probablemente esta tendencia se ha visto negativamente influida por el contexto pandémico, en el que esta modalidad de entrenamiento pueda despertar desconfianza por la interacción de un grupo de personas numeroso en espacios generalmente cerrados.

\section{¿Qué se incorpora en la encuesta de 2022?}

Las dos tendencias que se incorporan con respecto a 
la encuesta nacional de 2021 son el «entrenamiento del core»y la «tecnología portátil para vestir (wearables) relacionada con el deporte». Esta última es una tendencia con una gran relevancia en el contexto internacional que vuelve a aparecer en la encuesta española después de tres años fuera del top-20. El entrenamiento del core también reaparece en la encuesta nacional después de dos años de ausencia, mientras que esta tendencia ha perdido peso en el contexto internacional donde apareció por última vez hace 5 años (encuesta de 2018).

\section{Sobre el impacto del COVID-19 en la incor- poración de tendencias tecnológicas en el sec- tor del fitness}

En esta edición se ha intentado analizar el impacto del coronavirus en la incorporación de tecnología en el sector del fitness. Para ello, se elaboraron preguntas dirigidas a aquellos profesionales que permanecieron en el mismo centro deportivo durante el periodo de los dos últimos años y que, por tanto, podían tener una perspectiva de lo ocurrido durante la evolución de la enfermedad. Como se ha mostrado en la tabla 5, tras el estallido de la crisis sanitaria provocada por la pandemia de la COVID-19 se produjo un incremento en la existencia de sesiones de entrenamiento online en streaming, sesiones de entrenamiento online bajo demanda y de entrenamiento personal online. Los resultados muestran que después de la pandemia la oferta de estos tres servicios prácticamente se duplicó. Sin embargo, la utilización de apps para gestión de aforos se incrementó de forma importante pero mucho más moderada. Estos datos parecen indicar de forma bastante evidente que se ha incrementado la incorporación de tecnología en el sector del fitness durante la pandemia por parte de los proveedores de servicios (centros de fitness) lo cual, sin embargo, contrasta con el hecho de que ni las clases online ni el entrenamiento personal online aparezcan dentro del top-20 de la encuesta (posiciones \#34 y \#29). En cuanto al uso de wearables y apps por parte de los usuarios para controlar y guiar sus entrenamientos, los resultados obtenidos muestran que los encuestados perciben que su uso está poco extendido todavía. Estos resultados son congruentes con las posiciones alcanzadas por esas tendencias tanto en la encuesta española de este año como en las anteriores. En síntesis, los datos sugieren que la COVID-19 parece haber tenido un fuerte impacto en la adopción de la tecnología por parte de los prestadores de servicios, pero no tanto por parte de los usuarios de los servicios.

\section{Limitaciones $\mathrm{y}$ fortalezas}

Este estudio presenta las limitaciones propias de los estudios basados en encuestas en línea (Díaz de Rada, 2012), como son la falta de representatividad poblacional y las asociadas a la tasa de respuesta. No obstante, la tasa de respuesta conseguida en el presente estudio puede considerarse aceptable, alcanzando un 7.9\%, similar a la obtenida en las últimas tres encuestas nacionales de tendencias del fitness y superior a las de las ediciones de 2017y 2018.

Por otro lado, la principal fortaleza del estudio es que se replica la metodología de las encuestas internacionales del ACSM, permitiendo realizar comparaciones entre los datos nacionales e internacionales, tal y como se ha realizado durante este trabajo. Además, ésta constituye la sexta edición de las encuestas del fitness en España, país pionero en la replicación de las encuestas internacionales, lo que permite acumular resultados que permitan analizar la evolución de las tendencias a lo largo del tiempo mediante la comparación de las diferentes encuestas nacionales. Así mismo, dado que otros países también han empezado a replicar las encuestas en cada región específica siguiendo la misma metodología, la realización de análisis trasnacionales en el sector del fitness resultarán también posibles.

\section{Conclusiones}

A modo de conclusión puede decirse que los resultados de esta nueva edición de la encuesta española son bastante similares a los del año anterior y bastante discrepantes con los resultados de la encuesta internacional, tal y como se ha observado repetidamente en ediciones previas. La coincidencia entre ambas encuestas españolas se intensifica con relación a las primeras posiciones del ranking, así como también se intensifican las discrepancias en relación con las primeras posiciones de la encuesta internacional. Cabe destacar que esta pauta se viene observando año a año cada vez con más fuerza. Por otro lado, con relación al impacto de la COVID-19 en las tendencias tecnológicas los resultados muestran, como era de esperar, que ésta ha tenido un impacto relevante en la implantación de su uso por parte de los centros para la prestación de servicios a los usuarios, mientras que parece que todavía no está ampliamente extendido el uso de apps de entrenamiento y tecnología wearable entre los usuarios de los centros de fitness en España. 


\section{Referencias}

Amaral, P.C., \& Palma, D. D. (2019). Brazil and Argentina survey offitness trends for 2020. ACSM's Health \& Fitness Journal, 23(6), 36-40. https: / /doi.org/10.1249/FIT.0000000000000525

Batrakoulis,A. (2019). European survey of fitness trends for 2020. ACSM's Health \& Fitness Journal, 23(6), 28-35.https://doi.org/10.1249/ FIT.0000000000000523

Batrakoulis, A., Chatzinikolaou, A., Jamurtas, A. Z., \& Fatouros, I. G. (2020). National survey of fitness trends in Greece for 2021. International Journal of Human Movement and Sports Sciences, 8(6), 308-320. https: / / doi.org/10.13189/saj.2020.080602

Consejo COPLEF. (2019). Conoce las actuales leyes autonomicas de regulacion del ejercicio profesional del deporte. https://www.consejo-colef.es/ regulacion-autonomica

de la Camara, M.A.,ValcarceTorrente, M., \&Veiga, O. L. (2020). Encuesta nacional de tendencias defitness en España para el año 2020. Retos, 37, 434-441. https://doi.org/10.47197/retos.v37i37.74179

Díaz de Rada, V. (2012). Ventajas e inconvenientes de la encuesta por internet. Papers, 97(1), 193-223. https://doi.org/10.5565/rev/ papers/v97n1.71

Franco, S., Rocha, R. S., Ramalho, F., Simões,V., Isabel,V., \& Ramos, L. R. (2021). Tendências do Fitness em Portugal para 2021. Cuadernos de Psicología del Deporte, 21(2), 242-258. https://doi.org/10.6018/ cpd.467381

Gómez, L. F., López, J., Pelayo,A. R., \& Aguirre, L. E. (2020). Encuesta Nacional deTendencias Fitness para México en 2020 Retos, (39), 3037. https://doi.org/10.47197/retos.v0i39.78113

Kercher, V. M. (2018). International comparisons: ACSM's worldwide survey of fitness trends. ACSM's Health \& Fitness Journal, 22(6), 24-29. https://doi.org/10.1249/FIT.0000000000000431

Kercher,V.M., Kercher, K., Bennion,T.,Yates, B.A., Feito,Y.,Alexander, C., ... \& Miguel, Á. (2021). Fitness trends from around the globe. ACSM's Health \& Fitness Journal, 25(1), 20-31.https: / / doi.org/ 10.1249/FIT.00000000000000639

Kercher, V., Feito, Y., \& Yates, B. (2019). Regional comparisons: the worldwide survey offitness trends. ACSM's Health \& Fitness Journal, 23(6), 41-48.https://doi.org/10.1249/FIT.0000000000000531

Li, Y. M., Han, J., Liu,Y., Wang, R., Wang, R., Wu, X. P., \& Cao, Z. B. (2019). China survey of fitness trends for 2020. ACSM's Health \& Fitness Journal, 23(6), 19-27.https://doi.org/10.1249/ FIT.0000000000000522

Thompson, W. R. (2006). Worldwide survey reveals fitness trends for 2007. ACSM's Health \& Fitness Journal, 10(6), 8-14.https: / / doi.org/ 10.1249/01.FIT.0000252519.52241.39

Thompson, W. R. (2007). Worldwide survey reveals fitness trends for 2008. ACSM's Health \& Fitness Journal, 11(6), 7-13.https:/ / doi.org/ 10.1249/01.FTT.0000298449.25061.a8

Thompson, W. R. (2008). Worldwide survey reveals fitness trends for 2009. ACSM's Health and Fitness Journal, 12(6), 7-14.https: / / doi.org/ 10.1249/01.FTT.0000312432.13689.a4

Thompson, W. R. (2009). Worldwide survey reveals fitness trends for 2010. ACSM'S Health \& Fitness Journal, 13(6), 9-16.https: / / doi.org/ 10.1249/FIT.0b013e3181bcd89b

Thompson,W. R. (2010). Worldwide survey of fitness trends for 2011. ACSM's Health \& Fitness Journal, 14, 6-17.https://doi.org/10.1249/ FIT.0b013e3181f96ce6

Thompson, W. R. (2011). Worldwide survey of fitness trends for 2012. ACSM's Health \& Fitness Journal, 15(6), 9-18.https://doi.org/10.1249/ FIT.0b013e31823373cb
Thompson, W. R. (2012). Worldwide survey of fitness trends for 2013. ACSM' Health and Fitness Journal, 16(6), 8-17.https://doi.org/10.1249/ 01.FIT.0000422568.47859.35

Thompson, W. R. (2013). Now trending: worldwide survey of fitness trends for 2014. ACSM's Health \& Fitness Journalnal, 17(6), 10-20.https:/ /doi.org/10.1249/FIT.0b013e3182a955e6

Thompson, W. R. (2014). Worldwide survey of fitness trends for 2015. ACSM1/4s Health \& Fitness Journal, 18(6), 8-17. https://doi.org/ 10.1249/FIT.0000000000000073

Thompson, W. R. (2015). Worldwide survey of fitness trends for 2016: 10thAnniversary Edition. ACSM's Health and Fitness Journal, 19(6), 9-16. https://doi.org/10.1249/FIT.0000000000000164

Thompson, W. R. (2016). Worldwide survey of fitness trends for 2017. ACSM' Health and Fitness Journal, 2O(6), 8-17.https://doi.org/10.1249/ FIT.0000000000000252

Thompson,W.R.(2017).Worldwide survey of fitness trends for 2018:The CREP Edition. ACSM's Health \& Fitness Journal, 21(6), 11-19. https: / / doi.org/10.1249/FIT.0000000000000341

Thompson,W. R. (2018). Worldwide survey of fitness trends for 2019. ACSM's Health \& Fitness Journal, 22(6), 10-17. https: / / doi.org/https: / /doi.org/10.1249/FIT.0000000000000341

Thompson, W. R. (2019). Worldwide survey of fitness trend for 2020. ACSM'S Health\&FitnessJournal, 23(6), 10-18.https://doi.org/10.1249/ FIT.0000000000000526

Thompson, W. R. (2020). Worldwide survey of fitness trend for 2021. ACSM'S Health \& FitnessJournal, 25(1),10-19.https://doi.org/10.1249/ FIT.0000000000000621

Thompson,W. R., Sallis, R., Joy, E., Jaworski, C.A., Stuhr, R. M., \&Trilk, J. L. (2020). Exercise is Medicine. American Journal of Lifestyle Medicine, 14(5), 511-523.https://doi.org/ $10.1177 \% 2 \mathrm{~F} 1559827620912192$

Valcarce-Torrente, M., \& Garcia-Fernández, J. (2021). 3er Informe Impacto COVID-19 en las Instalaciones Deportivas. https: / / www.valgo.es/3erinforme-impacto-covid-19-en-las-instalaciones-deportivas

Valcarce-Torrente, M., Veiga, O. L., Arroyo-Nieto, Ángela, \& MoralesRincón, C. (2021). Encuesta Nacional de Tendencias de Fitness en Colombia para el Año 2021 (National Survey of Fitness Trends in Colombiafor 2021). Retos, 43, 107-116. https: / /doi.org/10.47197/ retos.v43i0.88528

Veiga, O. L., ValcarceTorrente, M., \& King Clavero,A. (2017). National survey of fitness trends in Spain for 2017. Apunts. Educacion Fisica $y$ Deportes, 128, 108-125. https://doi.org/10.5672/apunts.20140983.cat.(2017/2).128.07

Veiga, O.L.,ValcarceTorrente, M., \& King Clavero,A. (2018). Encuesta Nacional deTendencias de Fitness en España para 2018. Retos, 33, 279 285. https://doi.org/10.5672/apunts.2014-0983.es.(2017/ 2). 128.07

Veiga, O. L., Valcarce, M., King, A., \& de la Cámara, M. Á. (2019). Encuesta Nacional de Tendencias de Fitness en España para 2019. Retos, 35, 341-347. https://doi.org/https://doi.org/10.47197/ retos.v0i35.67353

Veiga, O., Valcarce-Torrente, M., \& de la Cámara, M. (2020). Encuesta Nacional deTendencias de Fitness en España para 2021. Retos, 39, 780-789. https: / / doi.org/10.47197/retos.v1i40.83008

Vorobiova,A. (2019).World and national fitness trends 2019. Sport Science and Human Health, 1(1), 10-17. https://doi.org/10.28925/26642069.2019.1.2 